\title{
Opportunities to Overcome Implementation Challenges of Infection Prevention and Control in Low-Middle Income Countries
}

Sharmila Sengupta, $M D^{1, *}$

Purabi Barman, $M D^{2}$ Jamie Lo, $M P H^{1}$

\author{
Address \\ *,1 Department of Infectious Diseases, National Center for Infectious Diseases, Tan \\ Tock Seng Hospital, 16 Jln Tan Tock Seng, Singapore, 308442, Singapore \\ Email: sharmila.sengupta1211@gmail.com \\ ${ }^{2}$ Department of Clinical Microbiology, BL Kapoor Hospital, Pusa Road, New Delhi, \\ 110005, India
}

Published online: 27 July 2019

(C) Springer Science+Business Media, LLC, part of Springer Nature 2019

This article is part of Topical Collection on Infection Prevention and Safety in Low and Middle Income Countries

Keywords Lowe-middle-income countries $\cdot$ Infection prevention and control $\cdot$ Opportunities

\begin{abstract}
Purpose of review Healthcare-associated infections (HAIs) and rising antimicrobial resistance (AMR) have posed a major challenge in patient care across the globe, more so in lower-middle-income countries (LMICs). Studies have shown that implementation of an effective infection prevention and control (IPC) program reduces incidence of HAI to a large extent. However, implementation of such a program in low-resource setting has many challenges. Research have shown that gaps exist due to absence of political will, trained manpower, and alternate healthcare priorities.

Recent findings With progress in science of healthcare epidemiology and digital communication, opportunities to establish an effective IPC program at minimal cost is possible. In this review, we explore ways the existing challenges can be mitigated in LMICs. In recent times, the UN general assembly along with World Health Organization (2015) and member countries took a resolution to come up with an action plan to tackle the rising threat of AMR by strengthening IPC programs at national level.
\end{abstract}


Summary We reviewed the progress made by the developing countries in their efforts to implement the program.

\section{Introduction}

Across the globe, healthcare-associated infections (HAIs) pose a major concern in all healthcare systems. It contributes significantly to patient morbidity and mortality particularly in low- and middleincome countries (LMICs). The prevalence is generally higher $(>40 \%)$ than that in high-income countries (HICs) (3.5-12\%) and has major economic consequences [1].

"Low-middle income countries" are classified by the World Bank according to their economies and Gross National Income (GNI) per capita (World Bank Atlas method). For the current 2019 fiscal year, low-income economies are those with a GNI per capita of $\$ 995$ or less in 2017; lowe-middle-income economies are those with a GNI per capita between $\$ 996$ and \$3895 [2]. LMICs include about 147 countries in Latin America, Sub-Saharan Africa, South Eastern Europe, and major parts of Asia-Pacific (barring countries such as Japan, Taiwan, South Korea, Singapore, Australia, and New Zealand) [3].
The magnitude of the problem remains underestimated or largely unknown because diagnosis is complex and surveillance activities to guide interventions are minimal in these countries. The most recent estimates in developing countries found the prevalence of HAI to be 15.5 per 100 patients (95\% CI 12.6-18.9) [4]. A survey conducted by the World Health Organization (WHO) in 2010 states that only $15.6 \%$ of developing nations reported a functioning national surveillance system. Intensive care unit (ICU)-acquired infections were as high as 35.2\% (95\% CI 24.2-48.0) (pooled cumulative incidence) and device-associated infection densities are up to 13 times higher than those in developed countries like the USA [5]. Gap analysis of infection prevention and control (IPC) practices across six international sites in LMICs shows adherence to recommended practices is suboptimal. Opportunities for improvement exist in several areas including regulatory mechanisms, guidelines and policies, hospital wide IPC programs, surveillance, antibiotic stewardship, and improved hand hygiene [6].

\section{Current challenges in reducing healthcare-associated infections in low- and middle-income countries}

Few studies have addressed the gaps existing for accomplishing a strong foundation to reduce risks and spread of healthcare-associated infections $[7 \bullet, 8,9]$. The common challenges such as competing political agendas, resource constraints, lack of trained personnel, and lack of surveillance data have always remained a barrier. Additionally, despite strong evidence for the effectiveness of IPC, further research is needed to identify and validate innovative technologies, cost-effectiveness of interventions, feasible implementation approaches and local solutions for low-resource settings [10].

Surveillance of healthcare-associated infections (HAIs) is the cornerstone of an effective infection prevention program. However, surveillance systems are almost non-existent in LMICs because of social and healthcare system deficiencies. Additionally, overcrowding, understaffed hospitals, inadequate IPC practices, lack of policies/guidelines and trained professionals add to the extent of the problem [11]. Healthcare workers (HCWs) lack knowledge regarding safe 
practices, risk of transmission of HAI, and hospital waste management [12].

Antimicrobial resistance (AMR) is not a recent phenomenon but has become a critical threat to the human population. A recent development, and cause of concern, is an apparent shift in the burden of AMR occurring between the main classes of pathogens from Gram-positive to Gram-negative bacteria [13] and Candida auris-an emerging multidrug-resistant yeast that can cause invasive infections, which is associated with high mortality, and spreads in healthcare settings [14]. This could rather stretch the already limited resources of health services as resistant Gram-negative pathogens will soon outweigh the achievements in reduction of resistant Gram-positive pathogens. Carbapenem-resistant Enterobacteriaceae (CRE) has left very few options for treatment. Colistin, which is considered as the "last resort" drug in CRE, has also shown resistance in clinical isolates. Reports from Greece, Italy, China, Turkey, and India are documented evidence [15-20]. Recent study published from India finds high levels of mortality due to colistin-resistant bacterial infections [21]. Lack of antibiotic policy, self-medication, over the counter availability of cheap substandard, counterfeit antimicrobials, and unregulated usage all contribute to prevalence of resistance in these countries. Maximum numbers of counterfeit drugs are found in Asia and Africa [22-24]. All these factors together contribute to the rising and spread of AMR across the world.

\section{Available opportunities to improve infection prevention and control in low-middle-income countries}

Prevention and control of HAI is an increasingly important element in improving healthcare more so in LMICs. Here, we would like to discuss the opportunities which are available to resource-limited settings, for implementation of an IPC program which includes antimicrobial stewardship.

It is to be noted that effective infection control does not require expensive resources and can be implemented with minimal cost $[25,26]$. It is just as feasible to institute appropriate IPC practices in low-resource settings as in highresource settings. It requires strategic approach, clinical leadership, and administrative will, and is based on commonsense, sound knowledge of procedures, and safe practices.

The core components of IPC as categorized by the WHO are applicable to most healthcare settings [27•]. It is multidisciplinary and multi-modal but inter-related collectively, and can be woven into a network of hospitals, regions, and countries both in high- and low-resource settings, thus adding value to

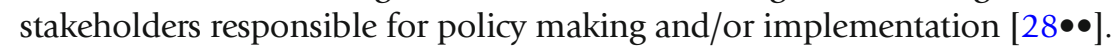

1. IPC program

2. IPC guidelines

3. IPC education and training 

4. Surveillance
5. Multimodal strategies
6. Monitoring/audit of IPC practices and feedback
7. Workload, staffing, and bed occupancy (acute healthcare facility only)
8. Build environment, materials, and equipment for IPC at the facility level (acute healthcare facility only)

The first step in this direction is planning an infection prevention and control (IPC) program. IPC will vary according to the size and scope of the organization. There are three essential and fundamental concepts to the program: (i) mission statement which is the reflection of the needs to be addressed by the program; (ii) vision which describes the future goals of the IPC program for the organization; and (iii) core values that serve as the blueprint of the program and indicate how the program functions on daily basis [29].

The electronic media and satellite communication has been a great boon to healthcare in this millennium. A large body of resources and knowledge on infection prevention are available and accessible through the internet. The evidences from studies done in developed countries such as the USA (CDC, NHSN, AHRQ, IHI), the UK (NICE guidelines), EU, and Australia are available and can be adapted and customized to LMICs. The basic principles of infection prevention being the same, the applicability of all methods maybe difficult in resource-limited settings, where innovation is the key.

Multiple tools have been developed for the assessment of infection control by the WHO and the USAID (Agency for International Development). The Infection Control Assessment Tool (ICAT) aims to identify targets for improved IPC practice which can be accessed easily [30]. However, it should be remembered that standards set by developed countries are often unachievable in LMICs due resource limitations, institutional priorities, and lack of expertise [31]. More studies need to be conducted, to ascertain appropriate, available, accessible, affordable, and applicable tools for lowresource settings to bridge the existing gap.

IPC education, training, and research

The presence of policies and guidelines does not necessarily ensure implementation in resource-limited settings. Introduction of structured curricula in medical and nursing education and in-house training of healthcare workers (HCWs) through workshops and CMEs will help in capacity building [32-34]. An area of opportunity lies in translation of international guidelines into simple local languages, which can be followed by groundlevel HCWs in their day-to-day practices. Utilization of communication technology is an easy and affordable way to disseminate knowledge on IPC 
and AMR among HCWs, patients, and public.

Research requires funding and expertise. Funding of research by highincome countries in LMICs will be a good opportunity to curb infectious diseases, which knows no borders [35]. There have been a good number of studies to show the cost-benefit of an IPC program in high-income countries [36]; however, such studies are required in low-resource settings. LMICs offer great potential for research in exploring epidemiology, pathogenesis, transmission, prevention, and economics of HAI. Among its efforts in this field, the WHO coordinates the Global IPC (GIPC) Network, which brings together major IPC organizations with the aim of enhancing local, national, and international collaboration [10]. HAI surveillance and sharing of data remain key issues for infection prevention and control program as have been proved since 1985 by the SENIC study [37] and confirmed by recent reviews [38]. An option for resourcelimited settings is implementation of periodic point prevalence survey (PPS) [39] compared to incidence studies, which require many more resources. PPS allows researchers to survey larger numbers of hospitals, even when human resources are limited. The existing gap of incidence studies which remain a reference for HAI description and understanding, the existing gap can be partially controlled by PPS repeated at defined intervals. The European Centre for Disease Prevention and Control (ECDC), within the framework of the "Antimicrobial resistance and healthcare-associated infection" program, developed and implemented multiple surveillance networks (starting from 2011 to 2012), conducting PPS for the detection of HAIs and antimicrobial use (AU) in acute hospitals [40]. A recent PPS study done in Italian hospitals shows this approach, guarantees a benchmark, and gives to the single hospital the opportunity to further improve its IPC program [41•]. Designing appropriate focus area of surveillance can be customized according to the individual healthcare settings and available resources following standard guidelines. In addition, surveillance information systems allow better "antibiotic resistance monitoring" and "antimicrobial use" helping to provide evidence-based results that can be used for the development of policies [42].

The microbiology laboratory plays a key role in success of a strong IPC by making data accessible. The design of the laboratory's record-keeping system if developed in collaboration with the infection control team accommodates the needs of the IPC program. Surveillance data should be regularly reported and shared at the regional and global levels [43]. There is also an urgent need to build laboratory capacity for ensuring reliable and rapid test results. Based on which, prescribing decisions and infection prevention measures can be taken. The use of technology such as electronic health records (EHR) and free microbiology software such as WHONET is great boon to LMICs where dearth of trained manpower is a formidable hindrance [44]. Basic hospital laboratories should be able to conduct appropriate sampling of specimens, culture for identification, and susceptibility for immediate patient care. Linking reference laboratories for epidemiological surveillance is a possible method to overcome high-end 
molecular testing. This enables obtaining reports on prevalence and spread antimicrobial resistance, detection of outbreaks, use of quality surveillance data for continuous improvement [45]. Point of care test (POCT) services can be used. These are low-cost tests, which do not need sophisticated instruments or skilled manpower and are easy to operate with a rapid turnaround time [46].

The scope of accreditation by national/international bodies also gives an opportunity to develop the IPC program, as it is one of the essential components of accreditation. Studies have shown accreditation to be positively associated with the establishment of organizational structures and processes, promotion of quality and safety cultures, improvements in patient care, and professional development being key benefits [47]. Unlike high-income countries where accreditation of healthcare is mandatory, many healthcare institutions in developing countries opt for the recognition through accreditation. This gives a competitive edge over other nonaccredited hospitals in terms of profit and attracting paying patients. The WHO's proposal for making the IPC a multi-modal, multi-disciplinary program enables low-resource settings to be more flexible and encourage

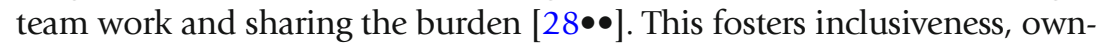
ership and sense of responsibility, and scope to all healthcare workers to participate and make it successful [48].

Monitoring/audit of IPC practices and feedback

Monitoring, audit, and feedback are important tools to spread awareness among healthcare workers, in achieving attitudinal change, besides engaging stakeholders in creating working partnerships in improving patient services. Audit and feedback generally lead to small but potentially important improvements in professional practice [49]. A feedback and feedforward cycle is a major tool for positive reinforcement and encourages healthy competition and active participation of individuals, within healthcare organizations.

Workload, staffing and bed occupancy (acute care facility only)

The WHO workload indicators and staffing needs provides a guide to healthcare managers in planning and distributing workload according to available manpower and resources [50]. Leaders of health systems must take the initiative to maximize the effectiveness of these programs by aligning them and funding them adequately [51•]. Ushering a culture change is more of perseverance and examples set by leaders in the organization and does not require heavy investments. Identifying motivated persons and empowering them to run the program lie in the hands of the administration. The challenge is in taking advantage of communication technology to train identified healthcare workers in the implementation of policies. Most important of all is to bring in a positive change of attitude and behavior among all HCWs towards the concept of prevention of HAI. Increase in hand hygiene compliance is the best example of bringing change in behavior of healthcare workers through perseverance and positive feedback [52]. 
Motivated key individuals if identified as champions can play a significant role in bringing change. Active champions influence organizational change through four functions: (i) building organizational support for new practices; (ii) protecting those involved in implementation from organizational rules and systems that may be barriers; (iii) helping to access the use of organizational resources for implementation; and (iv) supporting the growth of organizational coalitions to achieve implementation [53]. Champions are often well respected within organizations for their knowledge, skills, and interpersonal styles, and have the capacity to influence both the administration and the ground-level healthcare workers [54].

Build environment, materials and equipmentsfor IPC at the facility level (acute care facility only)

Identifying areas of high-risk for infections within the healthcare (e.g., OTs, ICUs, Labs) and providing with basic infrastructure, equipment, and materials such as hand hygiene products, masks, gloves, and gowns also promote a change in culture towards safe practices. Protocols for appropriate use need to be in place for better utilization and prevention of misuse and wastage $[55,56]$.

\section{Recent global efforts to facilitate infection prevention and antimicrobial resistance}

The UN General Assembly along with representatives from the WHO and representatives from member countries took a resolution on tackling the rising threat of antimicrobial resistance and urged to come up with an action plan by 2017-2018. Implementation of IC practices and antimicrobial stewardship in humans, animals, food, and agriculture (One Health) is essential for achieving the goal. Countries reaffirmed their commitment to develop national action plans on AMR (Fig. 1). Based on the "Global Action Plan on Antimicrobial Resistance" - the blueprint for tackling AMR developed in 2015 by the WHO [57]. Since then, many countries have attempted to establish IPC programs with varying degrees of success $[58,59]$. Countries like India with high burden of antimicrobial resistance have committed to come up with a national action plan [60-62]. Other initiatives, such as the Global Antibiotic Resistance Partnership (GARP) and the Global Health Security Agenda (GHSA), also aim to assess and improve national capacity for combatting AMR. GARP, a project of the Center for Disease Dynamics, Economics \& Policy (CDDEP), supports local creation of AMR policy by providing guidance, tools, and technical support to local researchers and policymakers, and is currently functioning in several LMICs $[63,64]$.

Latest update from the World Health Assembly (23 May 2019) says that "Member states agreed a resolution calling for high-level commitments to implement and adequately resource multi-sectorial National Action Plan to strengthen infection prevention and control measures including water 


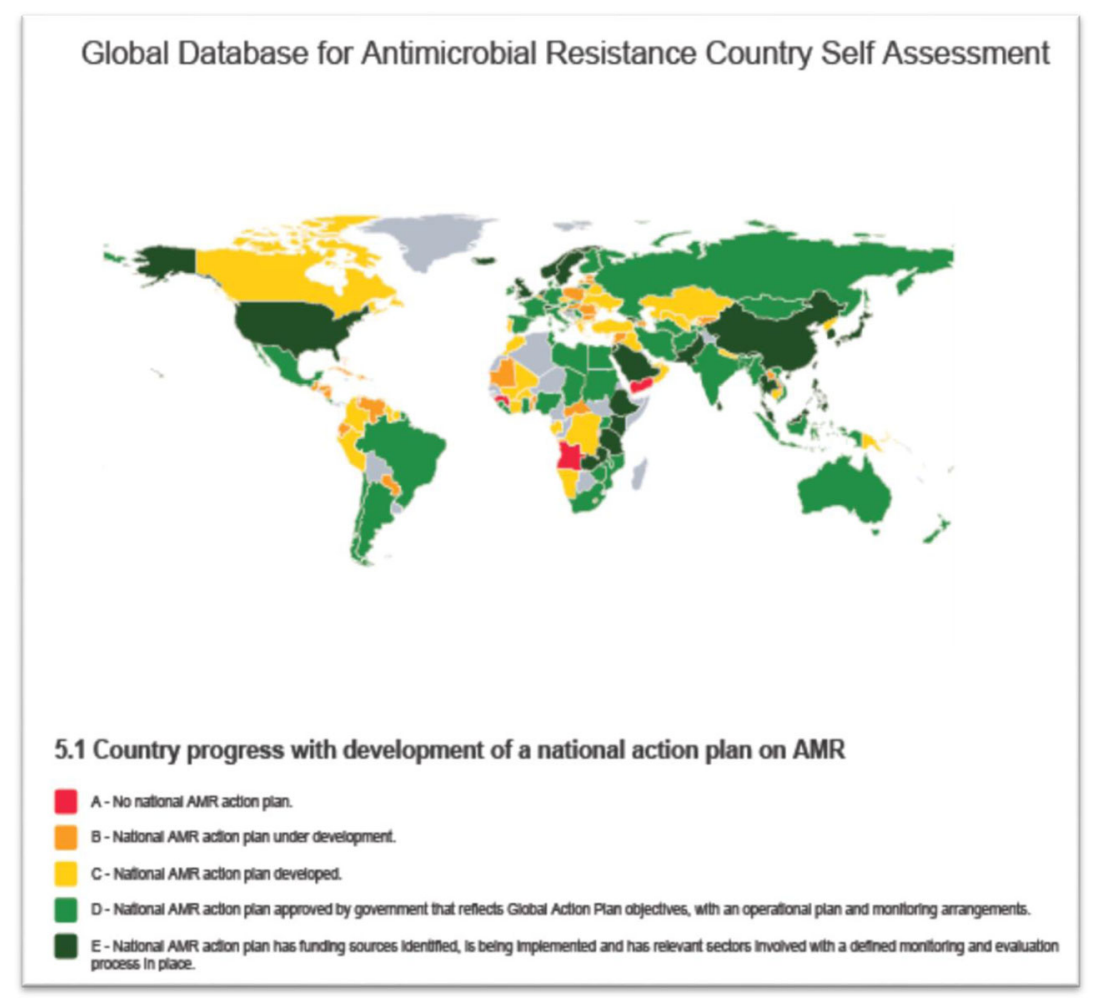

Fig. 1. Country progress with development of national action plan on AMR (courtesy: WHO).

sanitation and hygiene; enhance participation in Global Antimicrobial Surveillance System (GLASS); ensure prudent use of quality-assured antimicrobials; and support multi-sectorial annual self-assessment survey" [65].

Events such as worldwide infectious disease outbreak of SARS/Mers-coV/ Ebola in recent years became an important impetus for authorities in identifying gaps and providing opportunity to start IPC programs in affected countries. Such incidence has seen to trigger planning and implementation of IPC at national levels, creating awareness in health ministries for allocation of funds, leading to development of policies, improvement of infrastructure, and education of healthcare workers resulting in safer healthcare practices $[66,67]$.

\section{Ongoing efforts in infection prevention in LMICs}

\section{Latin America}

A recent study of 8 Latin American countries showed prevalence of patients with HAI was $11.6 \%$ (90/771; 95\% CI 9.3-14). Of all HAIs, 67\% were VAP, $18 \%$ were bloodstream infections, $13 \%$ were SSI, and $10 \%$ were UTI.

Similar prevalence was observed in other Latin American ICUs (27\% and 23.2\% in Argentina and Mexico, respectively). However, lower observed rates in the study might be due to lower sensitivity of diagnosis of infections made by sometimes insufficiently trained ICPs [68]. Countries like Chile and Venezuela have had ministry of health regulations since 1982 and 
1984; however, the implementation has been suboptimal, whereas there is good surveillance system in the hospital environment in Jamaica and the Caribbean islands [69].

IPC in these countries is in midst of transition. Countries such as those formerly in the United Soviet Socialist Republic (USSR), Mongolia, and post-conflict Eastern Europe are in their first stages of reform. Poor commitment, resource scarcity, and shortages of expertise exist. Underreporting of official infection control statistics is widespread. An example is in in Mongolia (located between Russia and China) wherein the Government approved a national program on sentinel surveillance system for HAIs in 2002 but implementation has been delayed due to insufficient support from stakeholders and shortages of resources and trained infection control professionals [70].

Egypt

Sub-Saharan Africa

Egypt established a national infection control program in 1999 [71]. During the last decade, while IPC activities were progressing in Egypt, a plan to implement a nationwide HAI surveillance program in intensive care units (ICUs) was developed with support from several partners: the U.S. Centers for Disease Control and Prevention's (CDC's) Global Disease Detection (GDD) Program in Egypt, the U.S. Naval Medical Research Unit (NAMRU3), and the U.S. Agency for International Development in Egypt. The target was to implement the surveillance program in all hospitals with ICUs before September 2018. Implementation of a sustainable surveillance system in a resource-limited country was possible following a stepwise approach with continuous evaluation. Enhancing IPC programs is now an infection control priority in Egypt [72].

Very little information is available on the epidemiology of HAI in African countries. The overall prevalence ranged from 2.5 to $14.8 \%$ (up to twice as high as the average European prevalence of $7.1 \%$ ). The pooled cumulative incidence and density of ICU-acquired HAIs were 34.7\% (95\% CI 23.647.7) and 47.9 per 1000 patient days (95\% CI 36.7-59.1), respectively. The real burden of HAIs is likely to be even greater in settings with weaker infrastructures and fewer resources. Most of the data available are from surveys conducted in university teaching hospitals which function as referral hospitals. The data available from the continent does not provide a comprehensive picture of the overall burden of HAI; instead, it provides the best overview possible while highlighting the many existing gaps [73].

South and Southeast Asia

Data from Asia are mostly isolated and limited to one or few hospital data, not a representation of the health systems of the country. The International 
Nosocomial Infection Control Consortium (INICC) network which has data of ICUs from the developing world also demonstrates value which is two- to threefold higher than ICUs in high-income countries. As a part of this network, there is a study of four hospitals in China [74] and seven hospitals (12 ICUs) from India. However, the study also claims that data may not accurately reflect the clinical setting of the country, and variations regarding surveillance methods may have affected the HAI rates [75]. A systematic review and metaanalysis of the burden of HAI in Southeast Asia revealed a pooled prevalence of overall HAI to 9\% [76]. The data contributed by smaller Asian countries like Philippines, Pakistan, and Thailand only reiterate the fact that HAI rates are threefold higher than the USA and that too is an under-report [77]. Brazil, Cuba, Mexico, Thailand, and Egypt in recent times are examples of few countries in the developing world that have established a national surveillance program for HAI. In most of other LMICs, the path to reducing the burden of HAI is still a challenge [4].

\section{Conclusion}

The future of patient safety is a responsibility of the medical community across the globe. The burden of HAI and AMR can be significantly reduced in the developing world through support of international organizations (WHO), agencies (CDC, ECDC), and professional societies (e.g., SHEA, APIC, IFIC, APSIC, HIS) and commitment from governments. The knowledge, research findings, and technical know-hows that are available could help in adaptation of suitable guidelines and customized to the needs of the developing nations. The WHO initiatives such as World Alliance for Patient Safety have become a global campaign. Its creation has helped to coordinate, spread, and accelerate improvements in reducing HAI [78]. It is well recognized that the problem of HAI is multidisciplinary, multi-organizational, and multinational. An effort to reduce the burden therefore needs a multi-prong approach of industrialized and developing nations working together towards patient safety.

\section{Compliance with Ethical Standards}

\section{Conflict of Interest}

Sharmila Sengupta declares that she has no conflict of interest.

Purabi Barman declares that she has no conflict of interest.

Jamie Lo declares that she has no conflict of interest.

Human and Animal Rights and Informed Consent

This article does not contain any studies with human or animal subjects performed by any of the authors. 
Papers of particular interest, published recently, have been highlighted as:

- Of importance

- Of major importance

1. Raka L. Lowbury lecture 2008: infection control and limited resources - searching for the best solutions. J Hosp Infect. 2009;72:292-8.

2. https://datahelpdesk.worldbank.org/knowledgebase/ articles/906519-world-bank-country-and-lendinggroups (Accessed 6 June 2019).

3. Bank W. World Bank country and lending groups: country classification. 2017 https://datahelpdesk. worldbank.org/knowledgebase/articles/906519world-bank-country-and-lending-groups (Accessed 6 June 2019).

4. Allegranzi B, Bagheri Nejad S, Combescure C, et al. Burden of health-care-associated infection in developing countries: review and meta-analysis. Lancet. 2011;377(9761):228-41.

5. World Health Organization. (2011). Report on the burden of endemic health care-associated infection worldwide. World Health Organization. http://www. who.int/iris/handle/10665/80135 (Accessed 6 June 2019).

6. Weinshel K, Dramowski A, Hajdu A, Jacob S, Khanal B, Zoltán $\mathrm{M}$, et al. Gap analysis of infection control practices in low- and middle-income countries. Infect Control Hosp Epidemiol. 2015;36(10):1208-24.

7. $\quad$ Bardossy AC, Zervos J, Zervos M. Preventing hospitalacquired infections in low-income and middle-income countries. Infect Dis Clin N Am. 2016;30(3):805-18

This article stresses on the unique issues faced by lowmiddle-income countries in prevention of healthcareacquired infection. The existing gaps are well discussed with suggestions on a step-by-step process for implementation of an infection prevention and control program.

8. Manchanda V, Suman U, Singh N. Implementing infection prevention and control programs when resources are limited. Curr Treat Options Infect Dis. 2018;10:28-39.

9. Alp E, Cookson B, Erdem H, Rello J, Akhvlediani T, Akkoyunlu Y, et al. Infection control bundles in intensive care: an international cross-sectional survey in lowand middle-income countries. Jr Hosp Infect. 2019;101:248-56.

10. Allegranzi B, et al. Global infection prevention and control priorities 2018-22: a call for action. Lancet. 2017;5(12):e1178-80.

11. Borg MA. Prevention and control of healthcare associated infections within developing countries. Int J Infect Control. 2010;v6:i1.

12. Lee R. Occupational transmission of bloodborne diseases to healthcare workers in developing countries: meeting the challenges. J Hosp Infect. 2009;72(4):285-91.
13. Nordmann P, Naas T, Poirel L. Global spread of carbapenemase producing Enterobacteriaceae. EID. 2011;17(10):1791-8.

14. Forsberg K, Woodworth K, Walters M, Berkow EL Jackson B, Chiller T, et al. Candida auris: the recent emergence of a multidrug-resistant fungal pathogen. Med Mycol. 2019;57:1-12.

15. Tansarli GS, Papaparaskevas J, Balaska M, Samarkos M, Pantazatou A, Markogiannakis A, et al. Colistin resistance in carbapenemase-producing Klebsiella pneumoniae bloodstream isolates: evolution over 15 years and temporal association with colistin use by time series analysis. Int J Antimicrob Agents. 2018 Sep;52(3):397-403.

16. Principe L, Piazza A, Mauri C, Anesi A, Bracco S, Brigante $\mathrm{G}$, et al. Multicenter prospective study on the prevalence of colistin resistance in Escherichia coli: relevance of mcr-1-positive clinical isolates in Lombardy, Northern Italy. Infect Drug Resist. 2018;11:37785.

17. Li Y, Sun Q-l, Shen Y, Zhang Y, et al. Rapid increase in prevalence of carbapenem-resistant Enterobacteriaceae (CRE) and emergence of colistin resistance gene mcr-1 in CRE in a hospital in Henan, China. J Clin Microbiol. 2018;56(4):e01932-17.

18. Kalem F, Ergun AG, Ertugul O, et al. Colistin resistance in Carbapenem-resistant Klebsiella pneumonia strains. Biomed Res - India. 2016;27(2):368-72.

19. Aggarwal R, Rastogi N, Mathur P, Soni KD, Kumar S, Gupta A, et al. Colistin-resistant Klebsiella pneumoniae in surgical polytrauma intensive care unit of level-1 trauma center: first case series from trauma patients in India. Indian J Crit Care Med. 2018;22(2):103-6.

20. Singh S, Pathak A, Kumar A, et al. Emergence of chromosome-borne colistin resistance gene mcr-1 in clinical isolates of Klebsiella pneumoniae from India. Antimicrob Agents Chemother. 2018;62(2):e01885-17.

21. Kaur A, Gandra S, Gupta P, et al. Clinical outcome of dual colistin- and carbapenem-resistant Klebsiella pneumonia bloodstream infections: a single-center retrospective study of 75 cases in India. Am J Infect Control. 2017;45(11):1289-91.

22. Ozawa S, Evans DR, Bessias S, et al. Prevalence and estimated economic burden of substandard and falsified medicines in low- and middle income countries: a systematic review and meta-analysis. JAMA Netw Open. 2018;1(4):e181662.

23. Kelesidis T, Falagas ME. Substandard/counterfeit antimicrobial drugs. Clin Microbiol Rev. 2015;28(2):443-64. 
24. Shakoor O, Taylor RB, Behrens RH. Assessment of the incidence of substandard drugs in developing countries. Tropical Med Int Health. 1997;2:839-45.

25. Apisarnthanarak A, Fraser VA. Feasibility and efficacy of infection-control interventions to reduce the number of nosocomial infections and drug-resistant microorganisms in developing countries: what else do we need? Clin Infect Dis. 2009;48(1):22-4.

26. Gill CJ, Mantaring JB, Macleod WB, et al. Impact of enhanced infection control at 2 neonatal intensive care units in the Philippines. Clin Infect Dis.

2009;48(1):13-21.

27. Storr J, Twyman A, Zingg W, et al. Core components for effective infection prevention and control programmes: new WHO evidence-based recommendations. Antimicrob Resist Infect Control. 2017;6(6):1-1.

This article written by the WHO Guideline development group discusses the core components set by the WHO. There are 11 recommendations and 3 good practice statements with a summary of the supporting evidence.

28.• World Health Organization. Guidelines on core components of infection prevention and control programmes at the national and acute health care facility level. Geneva: World Health Organization; 2017. Available at: http://apps.who.int/iris/handle/10665/ 251730. (Accessed 6 June 2019).

This is the WHO Guidelines published in January 2017, which details the core components of infection prevention and control program. They are intended to support countries in the development of national protocols for IPC and AMR. They are applicable for any country and suitable to local adaptations.

29. Dhar S, Cook E, Oden M, Kaye KS. Building a successful infection prevention program. Key components, processes and economics. Infect Dis Clin N Am. 2016;30(3):567-89.

30. Huskins W, Soule B, O'Boyle C, et al. Hospital infection prevention and control: a model for improving the quality of hospital care in low- and middle-income countries. Infect Control Hosp Epidemiol. 1998;19(2):125-35.

31. Leu H-S. The impact of US- style infection control programs in an Asian country. Infect Control Hosp Epidemiol. 1995;16(6):359-64.

32. Singh SP, Modi C, Patel C, et al. Introduction of infection control model for undergraduate medical students: experience at a rural medical college in India. Int J Infect Control. 2014; v10:i3.

33. Koo E, McNamara S, Lansing B, Olmsted RN, Rye RA, Fitzgerald $\mathrm{T}$, et al. Making infection prevention education interactive can enhance knowledge and improve outcomes: results from the targeted infection prevention (TIP) study. Am J Infect Control. 2016;44(11):1241-6.

34. Massaroli A, Martini JG, Medina-Moya JL, Bitencourt JVOV, Reibnitz KS, Bernardi MC. Teaching of infection control in undergraduate courses in health sciences: opinion of experts. Rev Bras Enferm [Internet]. 2018;71(Suppl 4):1626-34.

35. Ferez P, Bonomo RA. Carbapenemase-resistant Enterobacteriaceae: global action required. Lancet Infect Dis. 2019;19(6):601-10.

36. Dick A, Perencevich EN, Pogorzeleska-Maziarz M, et al. A decade of investment in infection prevention: a costeffectiveness analysis. Am J Infect Control.

2015;43(1):4-9.

37. Haley RW, Culver DH, White JW, Morgan WM, Emori TG, Munn VP, et al. The efficacy of infection surveillance and control programs in preventing nosocomial infections in US hospitals. Am J Epidemiol. 1985;121(2):182-205.

38. Zingg W, Holmes A, Dettenkofer M, Goetting T, Secci F, Clack L, et al. Hospital organisation, management, and structure for prevention of health-care-associated infection: a systematic review and expert consensus. Lancet Infect Dis. 2015;15(2):212-24.

39. Pearce N. Classification of epidemiological study designs. Int J Epidemiol. 2012;41:393-7.

40. European Centre for Disease Prevention and Control. Point prevalence survey of healthcare-associated infections and antimicrobial use in European acute care hospitals ECDC, Stockholm, 2013. Available at: https://ecdc.europa.eu/sites/portal/files/media/en/ publications/Publications/healthcare-associatedinfections-antimicrobial-use-PPS.pdf, (Accessed 6 June 2019).

41. Arnoldo L, Smaniotto C, Celotto D, et al. Monitoring healthcare-associated infections and antimicrobial use at regional level through repeated point prevalence surveys: what can be learnt? J Hosp Infect. 2019;101(4):447-5.

This research article discusses the benefits of repeated point prevalence survey which helped in reducing the HAI prevalence over a period of 7 years. It also helped in keeping attention on HAIs and antimicrobial utilization through the years and helped in standardizing the method along with a comparative evaluation in all participating regional hospitals.

42. Singh SK, Sengupta S, Antony R, et al. Variations in antibiotic use across India - multicentre study through global point prevalence survey. J Hosp Infect. 2019 (article in press). https://doi.org/10.1016/j.jhin.2019. 05.014 .

43. Simões AS, Couto I, Toscano C, Gonçalves E, Póvoa P, Viveiros $\mathrm{M}$, et al. Prevention and control of antimicrobial resistant healthcare-associated infections: the microbiology laboratory rocks! Front Microbiol. 2016;7:855.

44. Stelling JM, O'Brien TF. Surveillance of antimicrobial resistance: the WHONET program. Clin Infect Dis. 1997;24(Suppl 1):S157-68.

45. Chua KB, Gubler DJ. Perspectives of public health laboratories in emerging infectious diseases. Emerg Microbes Infect. 2013;2:e37.

46. Gous N, Boeras DI, Cheng B, Takle J, Cunningham B, Peeling RW. The impact of digital technologies on 
point-of-care diagnostics in resource-limited settings. Expert Rev Mol Diagn. 2018;18(4):385-97.

47. Nicklin W, Fortune T, van Ostenberg P, et al. Leveraging the full value and impact of accreditation. Int J Qual Health Care. 2017;29(2):310-2.

48. Ara L, Bashar F, Tamal NKA, et al. Transferring knowledge into practice: a multi-modal, multi-centre intervention for enhancing nurses' infection control competency in Bangladesh. J Hosp infect. 2019;102:234-40.

49. Ivers N, Jamtvedt G, Flottorp S, et al. Audit and feedback: effects on professional practice and healthcare outcomes. Cochrane Database Syst Rev. 2012;6:CD000259.

50. WHO. Workload indicator and staffing needs a user's manual World Health Organisation, 2015 (Accessed 6 June 2019).

51. Manning ML, Septimus EJ, Dodds Ashley ES, et al. Antimicrobial stewardship and infection prevention leveraging the synergy: a position paper update. Am J Infect Control. 2018;46(4):364-.

This is an interesting article which elaborates the shared common goal of IPC program and antibiotic stewardship to keep patients safe and improve patient outcome, regardless where care is delivered. It also identifies the core elements of CDC for implementation in all types of healthcare setting.

52. Atkins L. Using the behaviour change wheel in infection prevention and control practice. J Infect Prev. 2016;17(2):74-8.

53. Murray E, Holmes A. Addressing healthcare-associated infections and antimicrobial resistance from an organizational perspective: progress and challenges. J Antimicrob Chemother. 2012;67(Suppl 1):i29-36.

54. Damschroder LJ, Banaszak-Holl J, Kowalski CP, Forman J, Saint S, Krein SL. The role of the "champion" in infection prevention: results from a multisite qualitative study. BMJ Qual Saf. 2009;18:434-40.

55. Allegranzi B, Pittet D. Healthcare-associated infection in developing countries: simple solutions to meet complex challenges. Infect Control Hosp Epidemiol. 2007;28(12):1323-7.

56. Aiken AM, Allegranzi B, Scott JA, Mehtar S, Pittet D, Grundmann H. Antibiotic resistance needs global solutions. Lancet Infect Dis. 2014;14:550-1.

57. Sixty eighth World Health Assembly. Resolutions and decisions Geneva 2015 WHA68/2015/REC/1.

58. Gelband H, Miller-Petrie M, Pant S, Gandra S, Levinson J, Barter D, et al. The state of the world's antibiotics. Washington D.C: Center for Disease Dynamics, Economics \& Policy; 2015.

59. Zimmerman PA, Yeatman $H$, Jones $M$, et al. Success in south pacific: a case study of successful diffusion of an infection prevention and control program. Healthcare Infect. 2015;20(2):54-61.

60. Dutta SS. India launches strategy to curb antimicrobial resistance. BMJ. 2017;357:j2049.

61. Kakkar M, colleagues. Antibiotic resistance and its containment in India. BMJ. 2017;358:j2687.

62. Swaminathan S, Prasad J, Dhariwal A, et al. Strengthening infection prevention and control and systemic surveillance of healthcare associated infections in India. BMj. 2017;385:j3768.

63. Seale AC, Gordon NC, Islam J, Peacock SJ, Scott JAG. AMR surveillance in low and middle-income settings a roadmap for participation in the global antimicrobial surveillance system (GLASS). Wellcome Open Res. 2017;2:92.

64. Gelband H. The evolution of the global antibiotic resistance partnership. AMR Control. 2016;31-2. https://resistancecontrol.info/wp-content/uploads/ 2016/09/31-31-Gelband.pdf. (Accessed 9 June 2019).

65. World Health Assembly Update 2019. World Health Organization. www.who.int/news-room/detail/23-052019-world-health-assembly-update

66. Society for Healthcare Epidemiology of America. New document guides hospitals in responding to infectious disease outbreaks: healthcare epidemiologists play key role in emergency preparedness and response. ScienceDaily, 2017.

67. Banach DB, Johnston BL, Al-Zubeidi D, et al. Outbreak response and incident management: SHEA guidance and resources for healthcare epidemiologists in United States acute-care hospitals. Infect Control Hosp Epidemiol. 2017;38(12):1393-419.

68. Curcio D. Prevalence of nosocomial infection in Latin American intensive care units. Int J Infect Control. 2011;v7:i4.039.11.

69. Sastry S, Masroor N, Bearman G, et al. The 17th International Congress on Infectious Diseases workshop on developing infection prevention and control resources for low- middle-income countries. Int J Infect Dis. 2017;57:138-43.

70. Ider B-E, Adams J, Morton A, et al. Infection control systems in transition: the challenges for post-Soviet Bloc countries. J Hosp Infect. 2012;80(4):277-87.

71. Taalat M, Kandeel A, Rassian O, et al. Evolution of infection control in Egypt: achievements and challenges. Am J Infect Control. 2006;34(4):193-200.

72. Taalat $\mathrm{M}$, et al. National surveillance of health careassociated infections in Egypt: developing a sustainable program in a resource-limited country. Am J Infect Control. 2016;44(11):1296-301.

73. Bagheri Nejad S, Allegranzi B, Syed SB, Ellis B, Pittet D. Health-care-associated infection in Africa: a systematic review. Bull World Health Organ. 2011;89(10):757-65.

74. Hu B, Tao L, Liu K, et al. Device-associated infection rates, device use, length of stay and mortality in intensive care units of 4 Chinese hospitals: International Nosocomial Infection Control Consortium (INICC) finding. Am J Infect Control. 2012;03.037:1-6.

75. Mehta A, Rosenthal VD, Mehta Y, et al. Deviceassociated nosocomial infection rates in intensive care units of seven Indian cities. Findings of the International Nosocomial Infection Control Consortium (INICC). J Hosp Infect. 2007;67(2):168-74.

76. Lin ML, Apisarntharanak A, Madriaga G. The burden of healthcare associated infections in South-east Asia: a systemic literature review and meta-analysis. Clin Infect Dis. 2015;60(11):1690-9. 
77. Rosenthal VD, Maki D, Rodrigues C, et al. Impact of International Nosocomial Infection Control Consortium (INICC) strategy on central line-associated bloodstream infection rates in the intensive care units of 15 developing countries. Infect Control Hosp Epidemiol. 2010;31(12):1264-72.

78. Patient Safety: Making health care safer. Geneva: World Health Organization; 2017. License: CC BY-NC-SA 3.0 IGO.

\section{Publisher's Note}

Springer Nature remains neutral with regard to jurisdictional claims in published maps and institutional affiliations. 